\title{
ESTACIONALIDAD DEL GASTO EN SALUD EN COLOMBIA, $2011-2015^{*}$
}

\author{
Recibido: 28 de febrero de 2017 - Aprobado: 24 de mayo de 2017 \\ DOI: $10.22395 /$ seec.v20n44a7 \\ Óscar Andrés Espinosa Acuña** \\ Daniel Andrés Pinzón Fonseca**
}

\section{RESUMEN}

En este artículo se evalúa si existe estacionalidad en el gasto agregado en salud en Colombia entre los años 2011 y 2015. Para ello, se construye el gasto en salud desde las cuentas financieras de las empresas prestadoras de los servicios de salud en los regímenes contributivo y subsidiado, con periodicidad trimestral. Luego se aplican diferentes técnicas de análisis estadístico para determinar si existe estacionalidad: i) Método gráfico y tasas de crecimiento, ii) Correlogramas; iii) Contraste no paramétrico de Kendall, iv) Análisis de regresión múltiple con inclusión de dummies estacionales, y v) Ajuste de procesos autorregresivos integrados de media móvil estacional. Los resultados evidencian la existencia de estacionalidad estadísticamente significativa solo para gasto en salud realizado por las empresas prestadoras del régimen subsidiado, en especial en el cuarto trimestre (octubre-diciembre) de cada año.

\section{PALABRAS CLAVE}

Gasto en salud; estacionalidad del gasto en salud; gasto público en salud; régimen contributivo y subsidiado en salud; Colombia.

\section{CLASIFICACIÓN JEL}

I10, I18, H51.

\section{CONTENIDO}

Introducción; 1. Revisión de literatura; 2. Datos a modelar; 3. Metodologías estadísticas para determinar estacionalidad; 4. Resultados cuantitativos; 5. Conclusiones; Bibliografía.

\footnotetext{
Artículo de investigación desarrollado desde la Oficina de Metodologías de Supervisión y Análisis del Riesgo de la Superintendencia Nacional de Salud, durante el segundo semestre del año 2016. No se contó con financiación para el desarrollo de este proyecto. Trabajo Premiado en el V Congreso de la Asociación Colombiana de Economía de la Salud (Acoes), realizado del 29 de noviembre al 1 de diciembre de 2016 en la ciudad de Bogotá, Colombia.

"* Economista y magíster en Ciencias Estadísticas, Universidad Nacional de Colombia, Bogotá, Colombia Director del Grupo de Investigación en Modelos Económicos y Métodos Cuantitativos (Imemc, clasificado por Colciencias en categoría B en 2015), Centro de Investigaciones para el Desarrollo (CID), Facultad de Ciencias Económicas, Universidad Nacional de Colombia, Bogotá, Colombia. Dirección: Carrera 30 \#45-03, Edificio 311, Facultad de Ciencias Económicas, Segundo Piso, Oficina Decanatura, Bogotá, Colombia. Correo electrónico: oaespinosaa@unal.edu.co

"** Economista, profesional en Finanzas y magíster en Economía, Universidad del Rosario, Bogotá, Colombia. Magíster en Health Economics, Policy and Law, Erasmus Univesiteit de Rotterdam, Róterdam, Países Bajos. Jefe de la Oficina de Metodologías de Supervisión y Análisis del Riesgo, Superintendencia Nacional de Salud, Bogotá, Colombia. Catedrático en temas de economía de la salud en Universidad Pontificia Javeriana, Universidad del Rosario y Universidad EAN. Correo electrónico: dpinzon@supersalud.gov.co
} 


\section{SEASONALITY OF EXPENSE IN HEALTH IN COLOMBIA, 2011-2015 ABSTRACT}

This article evaluates whether or not there was seasonality in the aggregate expense in health in Colombia between 2011 and 2015. For this purpose, expense in health is built from the financial accounts of healthcare service companies (contributory and subsidized regimes) on a quarterly basis. Then, different statistical analysis techniques are applied in order to determine if there is seasonality: i) Graphic method and growth rates; ii) correlograms; iii) Kendall non-parametric contrast; iv) multiple regression analysis with inclusion of seasonal dummies; and v) Adjustment of self-regressive integrated processes of seasonal mobile media. Results show the existence of statistically significant seasonality only for expense in health, performed by healthcare service companies (subsidized regime), especially during the fourth quarter (October-December) each year.

\section{KEY WORDS}

Expense in health; seasonality of expense in health; public expense in health; contributory and subsidized health regime; Colombia.

\section{JEL CLASSIFICATION}

I10, I18, H51.

\section{CONTENT}

Introduction; 1. Revision of literature; 2. Data to be modeled; 3. Statistical methodologies to determine seasonality; 4. Quantitative results; 5. Conclusions; Bibliography.

\section{SAZONALIDADE DO GASTO EM SAÚDE NA COLÔMBIA, 2011-2015 RESUMO}

Neste artigo, avalia-se se existe sazonalidade no gasto agregado em saúde na Colômbia entre 2011 e 2015. Para isso, constrói-se o gasto em saúde a partir das contas financeiras das empresas prestadoras dos serviços de saúde nos regimes contributivo e subsidiado, com periodicidade trimestral. Logo, aplicam-se diferentes técnicas de análise estatística para determinar se existe sazonalidade: a) método gráfico e taxas de crescimento; b) correlogramas; c) contraste não paramétrico de Kendall, d) análise de regressão múltipla com inclusão de dummies sazonais; e) ajuste de processos autorregressivos integrados de média móvel estacional. Os resultados evidenciam a existência de sazonalidade estatisticamente significativa somente para gasto em saúde realizado pelas empresas prestadoras do regime subsidiado, em especial no quarto trimestre (outubro-dezembro) de cada ano.

\section{PALAVRAS-CHAVE}

Gasto em saúde; sazonalidade do gasto em saúde; gasto público em saúde; regime contributivo e subsidiado em saúde; Colômbia.

\section{CLASSIFICAÇÃO JEL}

I10, I18, H51.

\section{CONTEÚDO}

Introdução; 1. Revisão de literatura; 2. Dados a modelar; 3. Metodologias estatísticas para determinar sazonalidade; 4. Resultados quantitativos; 5 . Conclusões; Bibliografia. 


\section{INTRODUCCIÓN}

La dinámica del gasto en salud de las empresas prestadoras de los servicios de salud (EPS) en Colombia desde el año 2004 se ha visto influenciada por diferentes factores para los regímenes contributivo y subsidiado'. El primer régimen se ha visto afectado por el importante crecimiento de los pagos a través del Fosyga ${ }^{2}$ por concepto de recobros a las EPS por medicamentos no plan obligatorio de salud $\left(\right.$ POS $^{3}$ y por el crecimiento de los afiliados (MinSalud, 2014).

Por su parte, el segundo régimen ha estado dinamizado por las decisiones de Gobierno de aumentar progresivamente la cobertura de afiliación a este régimen, así como por la igualación de los planes de beneficios en pro de lo dispuesto por la Sentencia T-760 de $2008^{4}$ de la Corte Constitucional. Para el primer lustro de la segunda década del siglo XXI, el gasto total en salud representó aproximadamente el $7 \%$ del producto interno bruto colombiano, 0,8\% más que lo registrado en el período 2001-2010 (MinSalud, 2014).

Este artículo tiene por objetivo determinar si existe un comportamiento estacional en el gasto en salud de las entidades prestadoras de los servicios de salud de los regímenes contributivo y subsidiado en el país. El análisis exploratorio de la estacionalidad ayuda a los hacedores de política a obtener información primordial sobre la identificación de patrones en el sector salud y/o pautas repetitivas en el comportamiento dinámico de algunos tipos especiales de cuentas financieras.

Así, en este trabajo se adopta la definición de estacionalidad de Box y Jenkins (1976), la cual indica que el efecto estacional implica que una observación de un trimestre en particular, por ejemplo el segundo trimestre (T2), está relacionada con las observaciones de los segundos trimestres previos.

1 El régimen contributivo se define como el agregado de normas que orienta y rige la afiliación de la población con capacidad de pago al Sistema General de Seguridad Social en Salud (SGSSS). Por otra parte, el régimen subsidiado, se define como el conjunto de reglas que orienta y rige la afiliación de la población más pobre y vulnerable (sin capacidad de pago) al SGSSS. Para mayor detalle se puede consultar la Ley 100 de 1993.

2 Fondo de Solidaridad y Garantía (Fosyga), es un fondo del Ministerio de Salud y Protección Social cuya función principal es administrar los recursos que están destinados a inversiones relacionadas con la salud.

3 El POS es el conjunto de servicios a los que los afiliados al sistema de salud tienen derecho en el régimen contributivo del SGSSS. Los medicamentos no POS son aquellos que no se incluyen o no son cubiertos por este plan y deben ser adquiridos o comprados por el usuario.

4 De acuerdo con Yamín y Parra (2010) luego de un análisis estructural de la problemática en el acceso a la salud en Colombia, la Corte Constitucional dictaminó un conjunto de órdenes dirigidas a resolver las fallas de regulación referentes a la sostenibilidad financiera del sistema de salud, su dinámica en el flujo de recursos, y la cobertura del mismo. 
El artículo está estructurado de la siguiente forma: en la primera sección se realiza una revisión de literatura. El segundo aparte expone la construcción de los datos, partiendo de los planes de cuentas de las EPS públicas y privadas. Las metodologías para determinar la existencia o no de estacionalidad y sus respectivos resultados estadísticos se presentan, respectivamente, en la tercera y cuarta secciones. En la última sección se concluye.

\section{REVISIÓN DE LITERATURA}

La estacionalidad del gasto en salud no ha sido muy estudiada en la literatura internacional; no obstante, los determinantes del gasto en salud sí han sido objetivo de múltiples investigaciones en diferentes países.

Este es el caso de los trabajos de Gerdtham et al. (1992), Hitiris y Posnett (1992), Hansen y King (1996), Albouy, Davezies y Debrand (2010) y Murthy y Okunade (2016), quienes principalmente se concentran en la relación ingreso-salud para algunos países de la Organización para la Cooperación y el Desarrollo Económico (OCDE), mientras que las investigaciones de Govindaraj, Chellaraj y Murray (1997), Samudram, Nair y Vaithilingam (2009), Tang (2009), Clements, Faircloth y Verhoeven (2007) y Ke, Saksena y Holly (2011) revisan el tema para países en desarrollo.

En términos generales, los estudios encuentran que, además de ser el ingreso un factor determinante en la demanda de servicios de salud, los factores institucionales y socio-demográficos (p. e. el nivel educativo, la situación económica de los padres y el poseer un empleo) juegan un papel fundamental. También se señala que el gasto gubernamental en salud y los gastos de bolsillo ${ }^{5}$ siguen distintos caminos, y que el ritmo de crecimiento del gasto en salud es diferente para cada país, en función de su nivel de desarrollo económico.

Ya en relación concreta con la estacionalidad del gasto en salud, aunque no existen estudios que revisen su comportamiento. Ferrand et al. (2011) estudian la evolución temporal del gasto de los programas de Medicaid en agentes antidepresivos en EE. UU. Para ello, estiman modelos autorregresivos integrados de media móvil (ARIMA) con intervenciones (en especial las relacionadas con la entrada de medicamentos de marca y genéricos y la publicidad directa al consumidor). Los

5 Según Pérez-Valbuena y Silva-Ureña (2015) los gastos de bolsillo se definen como los pagos directos hechos por las personas en bienes y servicios del sector salud, tales como medicamentos, hospitalizaciones, entre otros tópicos, que no son cubiertos por el sistema de salud, sin importar si se encuentran o no afiliados a algún régimen. En Latinoamérica se tienen los estudios sobre gasto de bolsillo de Castillo-Laborde y Villalobos (2013), para Chile, y Pérez-Valbuena y Silva-Ureña (2015), para Colombia. 
autores encuentran que la entrada de medicamentos genéricos no condujo a una reducción de los gastos generales, siendo estos últimos no estacionales.

Bajo otro tópico de estudio, Loh (2015) busca comprender mejor las tendencias y determinantes de las actividades de turismo de salud en Canadá entre los años 1970 y 2010. Para ello, sigue una metodología de regresiones auxiliares sobre los residuales (con la que se contrasta la existencia de quiebres estructurales) y modelos autorregresivos de media móvil con variables exógenas (ARMAX). Se encuentra que el turismo de salud aumenta cuando la inversión privada en instalaciones médicas disminuye, siendo una de las posibles causas de este cambio estructural el Acuerdo General sobre el Comercio de Servicios (AGCS), que entró en vigor en 1995.

El trabajo de Safaeian et al. (2015) tiene por finalidad evaluar el patrón de estacionalidad y los factores relacionados con la prescripción de antibióticos en Isfahan (Irán). A través de una encuesta transversal se aplican múltiples modelos de regresión logística. Esta investigación concluye la existencia de un pico estacional en otoño de las recetas de penicilinas y cefalosporinas.

Uno de los estudios más recientes es el de Albert et al. (2015), donde se quiere conocer si las estaciones climáticas afectan los gastos de atención médica (MCE) en las personas de avanzada edad en Holanda. Para la descomposición estacional y de tendencia los autores aplican el método de Loess, usando la prueba t de Welch. Se concluye que las estaciones climáticas sí afectan los gastos, dado que aumentan las tasas de mortalidad y de hospitalización.

Para Colombia, uno de los primeros análisis formales sobre el gasto en salud fue el de O’Meara, Ruiz y Amaya (2003); no obstante, contempla un análisis netamente descriptivo y no se tratan temas de estacionalidad en específico.

Por ende, el objetivo de este artículo se considera novedoso para la literatura colombiana.

\section{DATOS A MODELAR}

En este documento el gasto en salud se entiende como el monto asignado por las EPS (indiferente del régimen perteneciente) a los productos y a los servicios prestados durante el ejercicio de sus labores. Bajo tal concepto, para las estimaciones del gasto en salud en las empresas de carácter privado, teniendo como marco de referencia el Plan Único de Cuentas (PUC) dispuesto en la Resolución 4175 de 2014 de la Superintendencia Nacional de Salud, se tomaron las cuentas relacionadas en la tabla $1^{6}$.

6 Para una explicación detallada de cada subcuenta remitirse a la respectiva norma. 
Tabla 1. Cuentas seleccionadas del PUC de la Resolución 4175 de 2014 de la Superintendencia Nacional de Salud (para empresas privadas)

\begin{tabular}{|c|c|c|}
\hline $\begin{array}{l}\text { Cuenta a dos } \\
\text { dígitos }\end{array}$ & Cuenta a cuatro dígitos & Cuenta a seis dígitos \\
\hline \multirow{12}{*}{$\begin{array}{l}\text { N. } .^{\circ} \text { 61: Costo } \\
\text { de ventas y de } \\
\text { prestación de } \\
\text { servicios }\end{array}$} & $\begin{array}{l}\text { N. }{ }^{\circ} 6105: \text { Costos prestación de } \\
\text { servicios de salud }\end{array}$ & $\begin{array}{l}\text { No aplica, se toma la cuenta agregada a cuatro } \\
\text { dígitos. }\end{array}$ \\
\hline & \multirow{9}{*}{$\begin{array}{l}\text { N. } 6165: \text { Administración del } \\
\text { régimen de seguridad social en } \\
\text { salud-POS- }\end{array}$} & N. 616535 : Contratos de capitación \\
\hline & & N. ${ }^{\circ}$ 616540: Contratos por evento \\
\hline & & $\begin{array}{l}\text { № } 616542: \text { Contratos para actividades de promo- } \\
\text { ción y prevención }\end{array}$ \\
\hline & & N. ${ }^{\circ}$ 616565: Seguridad social en salud \\
\hline & & $\begin{array}{l}\text { N. } 616575: \text { Enfermedades catastróficas y enfer- } \\
\text { medades de alto costo) }\end{array}$ \\
\hline & & $\begin{array}{l}\text { N. 616585: Costos de prestación de servicios } \\
\text { adicionales de salud }\end{array}$ \\
\hline & & $\begin{array}{l}\text { N. 616587: Costos de prestación de servicios de } \\
\text { ambulancia por demanda }\end{array}$ \\
\hline & & $\begin{array}{l}\text { N. 616590: Provisión de servicios autorizados y } \\
\text { no cobrados }\end{array}$ \\
\hline & & N.. 616595: Otros costos operacionales \\
\hline & $\begin{array}{l}\text { N. }{ }^{\circ} \text { 6170: Administración del } \\
\text { régimen de seguridad social en } \\
\text { salud no POS }\end{array}$ & $\begin{array}{l}\text { No aplica, se toma la cuenta agregada a cuatro } \\
\text { dígitos. }\end{array}$ \\
\hline & $\begin{array}{l}\text { N. }{ }^{\circ} \text { 6175: Prestaciones econó- } \\
\text { micas. }\end{array}$ & $\begin{array}{l}\text { No aplica, se toma la cuenta agregada a cuatro } \\
\text { dígitos. }\end{array}$ \\
\hline
\end{tabular}

Fuente: elaboración propia

Por otra parte, para las empresas de carácter público se consideraron, del PUC estipulado en la Resolución 414 de 2014 de la Contaduría General de la Nación, los rubros presentados en la tabla $2^{7}$.

Para el régimen contributivo, se consideró la totalidad de las EPS (14) del Sistema General de Seguridad Social en Salud (SGSSS). En el régimen subsidiado se tomaron 25 entidades (las cuales representan más del 80\% de los gastos totales en salud de este régimen), entre ellas, cajas de compensación familiar, empresas solidarias de salud y empresas promotoras de salud autorizadas para administrar recursos del régimen subsidiado ${ }^{8}$ (se excluyen las EPS indígenas dado que sus sistemas de

7 Para una explicación detallada de cada subcuenta remitirse a la respectiva norma.

8 Según la Ley 1122 de 2007 (Capítulo IV Del aseguramiento, Artículo 14), todas estas entidades se agrupan bajo el nombre de Entidades Promotoras de Salud del régimen subsidiado. 
información no presentan una buena calidad). La periodicidad es trimestral, desde el primer trimestre (T1) del año 2011 hasta el cuarto trimestre (T4) del año $2015^{9}$. Estos valores monetarios fueron deflactados por el IPC salud con año base 2011 (información obtenida del Departamento Administrativo Nacional de Estadística -DANE-).

Tabla 2. Cuentas seleccionadas del PUC de la Resolución 414 de 2014 de la Contaduría General de la Nación (para empresas públicas)

\begin{tabular}{|c|c|c|}
\hline $\begin{array}{l}\text { Cuenta a dos } \\
\text { dígitos }\end{array}$ & $\begin{array}{l}\text { Cuenta a } \\
\text { cuatro dígitos }\end{array}$ & Cuenta a seis dígitos \\
\hline \multirow{12}{*}{$\begin{array}{l}\text { N. }{ }^{\circ} \text { 64: Costo } \\
\text { de operación } \\
\text { de servicios }\end{array}$} & \multirow{12}{*}{$\begin{array}{l}\text { N. }{ }^{\circ} \text { 6402: Ad- } \\
\text { ministración } \\
\text { de la seguri- } \\
\text { dad social en } \\
\text { salud }\end{array}$} & N. ${ }^{\circ}$ 640201: Contratos de capitación - Contributivo \\
\hline & & N. ${ }^{\circ}$ 640202: Contratos por evento - Contributivo \\
\hline & & N. ${ }^{\circ}$ 640203: Promoción y prevención - Contributivo \\
\hline & & N. $640205:$ Reaseguro enfermedades de alto costo - Contributivo \\
\hline & & N. ${ }^{\circ}$ 640206: Incapacidades - Contributivo \\
\hline & & N. ${ }^{\circ}$ 640207: Contratos de capitación - Subsidiado \\
\hline & & N. ${ }^{\circ}$ 640208: Contratos por eventos - Subsidiado \\
\hline & & N. ${ }^{\circ}$ 640209: Promoción y prevención - Subsidiado \\
\hline & & 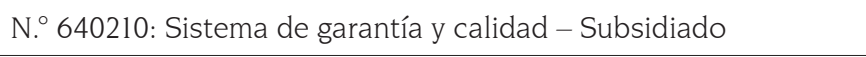 \\
\hline & & N. ${ }^{\circ}$ 640211: Reaseguro enfermedades de alto costo - Subsidiado \\
\hline & & N. ${ }^{\circ}$ 640212: Contratos de capitación - Complementario \\
\hline & & $\begin{array}{l}\text { N. } 640290 \text { : Otros costos por la administración de la seguridad } \\
\text { social en salud. }\end{array}$ \\
\hline
\end{tabular}

Fuente: elaboración propia

\section{METODOLOGÍAS ESTADÍSTICAS PARA DETERMINAR ESTACIONALIDAD}

Esta sección muestra las diferentes técnicas estadísticas aplicadas para el estudio del efecto de estacionalidad, como lo son el método gráfico, las tasas de crecimiento trimestre a trimestre, los correlogramas simples y parciales, la prueba no paramétrica de Kendall, el análisis de regresión con inclusión de variables dummies estacionales y los procesos estocásticos SARIMA $(\boldsymbol{p}, \boldsymbol{d}, \boldsymbol{q})(\mathbf{P}, \mathbf{S}, \mathbf{Q})_{\boldsymbol{s}}$.

9 La construcción de estos datos contempla las implicaciones relativas al Decreto 3797 de 2014 del Ministerio de Salud y Protección Social Por medio de la cual se asignan códigos a las Entidades Promotoras de Salud-EPS- y a las Cajas de Compensación Familiar-CCF-, para la operación de la movilidad. Los autores suministrarán la base de datos construida y utilizada en el estudio a cualquier investigador interesado, mediante solicitud al correo electrónico: oaespinosaa@unal.edu.co 


\subsection{Método gráfico y tasas de crecimiento trimestre a trimestre}

Este método a priori se fundamenta en encontrar de forma visual posibles patrones de estacionalidad al graficar la serie temporal de interés. Asimismo, se busca determinar a partir de las tasas de crecimiento simple si se evidencia algún comportamiento atípico cada $n$ períodos (cada 4 para periodicidad trimestral).

\subsection{Correlogramas simples (FAC) y parciales (FACP)}

Sea $\left\{Z_{t}\right\}$ un proceso estacionario con $E(Z t)=\mu$ y $\operatorname{Var}\left(Z_{t}\right)=\sigma^{2}$ constantes, se define la función de autocovarianza (FACV) como:

$$
\gamma_{k}=\operatorname{Cov}\left(Z_{t}, Z_{t-k}\right)=E\left(Z_{t}-\mu\right)\left(Z_{t-k}-\mu\right)
$$

La autocorrelación $\left(\rho_{k}\right)$ mide la relación (o dependencia, más no causalidad) entre dos variables separadas por $k$ períodos. De esta manera, la función de autocorrelación simple (FAC) se expresa mediante la ecuación [2].

$$
\rho_{k}=\operatorname{Corr}\left(Z_{t} Z_{t-k}\right)=\frac{\operatorname{Cov}\left(Z_{t}, Z_{t-k}\right)}{\sqrt[2]{\operatorname{Var}\left(Z_{t}\right)} \sqrt[2]{\operatorname{Var}\left(Z_{t-k}\right)}}=\frac{\gamma_{k}}{\gamma_{0}}
$$

Luego, todo proceso estacionario satisface las siguientes cinco características (Tsay, 2005) ${ }^{10}$ :

i) $\forall t, \gamma_{0}=\operatorname{Var}\left(Z_{t}\right) \cdot \forall t, \gamma_{0}=\operatorname{Var}\left(Z_{t}\right)$

ii) $\forall t, \rho_{0}=1$.

iii) $\forall k$, de $-1 \leq \rho_{k} \leq 1$, se tiene que $-\gamma_{0} \leq\left|\gamma_{k}\right| \leq \gamma_{0}$

iv) $\gamma_{k}$ y $\rho_{k}$ son simétricas con respecto a $k$, es decir, $\forall k \gamma_{k}=\gamma_{-k} \wedge \rho_{k}=\rho_{-k}$

v) $\gamma_{k}$ y $\rho_{k}$ son semidefinidas positivas. Es decir, se satisfacen las siguientes desigualdades:

$$
\forall t_{1}, \ldots, t_{n} ; \forall a_{1}, \ldots, a_{n}, \sum_{i=1}^{n} \sum_{j=1}^{n} a_{i} a_{j} \gamma_{\left|t_{i}-t_{j}\right|} \geq 0 \wedge \sum_{i=1}^{n} \sum_{j=1}^{n} a_{i} a_{j} \rho_{\left|t_{i}-t_{j}\right|} \geq 0
$$

Por otra parte, la autocorrelación parcial mide la dependencia entre $Z_{t}$ y $Z_{t-k}$ cuando se elimina el efecto de $Z_{t-1}, Z_{t-2}, \ldots, Z_{t-k+1}$. Se expresa mediante:

$$
\pi_{k}=\operatorname{Corr}\left(Z_{t}, Z_{t-k} \mid Z_{t-1}, Z_{t-2}, \ldots, Z_{t-k+1}\right)
$$

10 Adicionalmente, el valor del coeficiente de correlación no varía al hacerlo la escala de medición. 
Ahora, los correlogramas simples y parciales hacen referencia a una representación gráfica de las autocorrelaciones simples y parciales de la muestra, respectivamente, versus el tiempo. Si cada cierto número de período aumentan significativamente las autocorrelaciones, ello daría muestras de posibles estacionalidades.

\subsection{Prueba no paramétrica de Kendall}

Este contraste de estacionalidad basado en rangos, desarrollado por Kendall y Ord (1990), consiste en ordenar las observaciones de cada trimestre y asignarles un valor de 1 a la menor, hasta 4 a la mayor. A continuación, para cada trimestre se suman los valores de todos los años, por lo que:

$$
\mathrm{M}_{i}=\sum_{j=1}^{\tau} \operatorname{rank}\left(\text { trimestre }_{i}\right)_{j}
$$

Donde $\tau$ representa el número de años de la muestra. Así, bajo la hipótesis nula de no existencia de estacionalidad, el estadístico a calcular sigue la expresión:

$$
\mathrm{K}=\frac{4}{\tau r(r+1)} \sum_{i=1}^{r}\left(\mathrm{M}_{i}-\frac{\tau(r+1)}{2}\right)^{2}
$$

Donde $r$ es el número de observaciones por año (4 con datos trimestrales). Tal estadístico se distribuye como una $\chi^{2}$ con $(r-1)$ grados de libertad. Luego, para valores grandes de $\mathrm{K}$ (superiores al cuantil considerado de la $\chi^{2}$ ) se concluye que existe componente estacional.

\subsection{Análisis de regresión con inclusión de variables dummies estacionales}

En la regresión lineal múltiple de $\mathrm{Y}$ sobre $\mathrm{X}_{1}, \ldots, \mathrm{X}_{\mathrm{K}}$ se admite que la función de regresión tiene la expresión [6].

$$
\mathrm{E}\left(\mathrm{Y} \mid \mathrm{X}_{1}=x_{1}, \ldots, \mathrm{X}_{\mathrm{K}}=\chi_{\mathrm{K}}\right)=\beta_{0}+\beta_{1} \mathrm{X}_{1}+\ldots+\beta_{\mathrm{K}} \mathrm{X}_{\mathrm{K}}
$$

Al contemplar una muestra de $\mathrm{T}$ datos en los que se observa las variables $\mathrm{Y}$ y $\mathrm{X}$ $=\left(\mathrm{X}_{1}, \ldots, \mathrm{X}_{\mathrm{K}}\right)$ ' se tiene $\left(x_{t}, y_{t}\right), i=1, \ldots, t$, donde $x_{t}=\left(x_{t 1}, x_{t 2}, \ldots, x_{t k}\right)$. Luego, el modelo de regresión lineal múltiple asume que:

$$
y_{t}=\beta_{0}+\beta_{1} x_{t 1}+\ldots+\beta_{\mathrm{K}} x_{t K}+U_{t}, \quad t=1, \ldots, T
$$

Donde las perturbaciones $\mathrm{U}_{t}$ verifican las siguientes hipótesis:

i) $\mathrm{E}\left(\mathrm{U}_{t}\right)=0$, para cada $t=1, \ldots, \mathrm{T}$. 
ii) $\operatorname{Var}\left(\mathrm{U}_{t}\right)=\sigma^{2}$, para cada $t=1, \ldots, \mathrm{T}$

iii) $\mathrm{E}\left(\mathrm{U}_{t} \mathrm{U}_{i}\right)=0, \forall t \neq i$

iv) $U_{t} \sim \operatorname{Normal}\left(0, \sigma^{2}\right), \forall t$

v) $\mathrm{T} \geq \mathrm{K}+2$

vi) Las variables $\mathrm{X}_{t}$ son linealmente independientes entre sí (no hay colinealidad).

Así, para este estudio se plantean las ecuaciones [8] y [9].

$$
\begin{gathered}
\text { GSRCont }_{t}=\beta_{0}+\beta_{1} \text { CDSSRCont }_{t}+\beta_{2} \mathrm{D}_{\mathrm{Q} 2}+\beta_{3} \mathrm{D}_{\mathrm{O} 3}+\beta_{4} \mathrm{D}_{\mathrm{O} 4}+\mathrm{U}_{t} \\
t=1, \ldots, \mathrm{T} . \\
\text { GSRSubs }_{t}=\alpha_{0}+\alpha_{1} \text { CDSSRSubs }_{t}+\propto_{2} \mathrm{D}_{\mathrm{O} 2}+\propto_{3} \mathrm{D}_{\mathrm{Q} 3}+\alpha_{4} \mathrm{D}_{\mathrm{O} 4}+\mathrm{V}_{t} \\
t=1, \ldots, \mathrm{T}
\end{gathered}
$$

Donde GSRcont $t_{t}$ es el gasto en salud en el régimen contributivo en el período $t$; GSRSubs $_{t}$ es el gasto en salud en el régimen subsidiado en el período $t$; CDSSRCont $t_{t}$ es la cantidad demandada de servicios de salud en el régimen contributivo en el período $t$; CDSSRSubs $s_{t}$ es la cantidad demandada de servicios de salud en el régimen subsidiado en el períodot, y $\mathrm{D}_{\mathrm{Q} i}$ es una variable dummy que toma el valor de 1 cuando se está en el período trimestral $i$, con $i=2,3,4^{11}$.

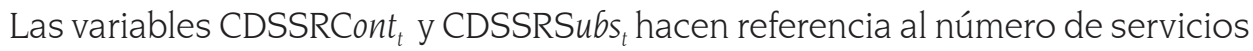
de demanda por hospitalización, medicamentos, nacidos, procedimientos y urgencias por cada régimen. Estos valores son tomados de la base de datos de Registros Individuales de Prestación de Servicios de Salud (RIPS), establecida por la Resolución 3374 de 2000 del Ministerio de Salud ${ }^{12}$.

Dada la estructura de la ecuación [7], en este sistema estadístico la estacionalidad se determina si al menos uno de los parámetros $\beta_{2}, \beta_{3}$ y $\beta_{4}\left(\propto_{2}, \propto_{3}\right.$ y $\left.\propto_{4}\right)$ es estadísticamente significativo. Y el parámetro $\beta_{1}\left(\propto_{1}\right)$ se puede interpretar como una variable control.

\footnotetext{
${ }^{11}$ Para esta variable dicotómica se tiene como referencia a Tl (variable dummy que indica cuando es primer trimestre) para evitar el problema de multicolinealidad.

${ }^{12}$ Los RIPS se definen como el conjunto de datos mínimos y básicos que el Sistema General de Seguridad Social en Salud requiere para los procesos de dirección, regulación y control, y como soporte de la venta de servicio.
} 


\subsection{Procesos estocásticos SARIMA $(p, d, q)(P, D, 0)_{s}$.}

Es considerado uno de los mejores modelos lineales para ajuste de series económicas, por su estructura dinámica constituida en la búsqueda de patrones históricos, con la premisa de que las futuras estimaciones solo dependen del pasado de la serie (de su inercia) y no de variables independientes (Brockwell y Davis, 2002). En la literatura este tipo de proceso estocástico se considera por excelencia uno de los mejores métodos para representar los componentes estacionales.

Adentrándose al aspecto formal, este modelo captura la característica estacional y cíclica de la serie de interés, entendiendo por cíclica la asociada al componente autorregresivo y de media móvil. Se expresa como sigue en [10].

$$
\begin{gathered}
\Phi(\mathrm{L}) \Phi_{s}(\mathrm{~L}) \nabla_{12}^{\mathrm{D}} \nabla_{1}^{d} \mathrm{Z}_{t}=\Theta(\mathrm{L}) \Theta_{s}(\mathrm{~L}) \varepsilon_{t} \\
\left(1-\sum_{k=1}^{p} \phi_{k} \mathrm{~L}^{k}\right)\left(1-\sum_{k=1}^{\mathrm{P}} \phi_{k}^{s} \mathrm{~L}^{k}\right)\left(1-\mathrm{L}^{s}\right)^{\mathrm{D}}(1-\mathrm{L})^{d} \mathrm{Z}_{t}=\left(1-\sum_{k=1}^{q} \theta_{k} \mathrm{~L}^{k}\right)\left(1-\sum_{k=1}^{\mathrm{O}} \theta_{k}^{s} \mathrm{~L}^{k}\right) \varepsilon_{t}
\end{gathered}
$$

Donde $\Phi(\mathrm{L})$ es el polinomio de coeficientes, con operador de rezagos $\mathrm{L}$ del componente autorregresivoAR; y $\Phi_{s}(\mathrm{~L})$ el símil al caso anterior, pero aplicado al proceso autorregresivo de la parte estacional SAR. Además, $\Phi(\mathrm{L})$ y $\Phi_{s}(\mathrm{~L})$ son los polinomios de coeficientes del componente de media móvil MA y su análogo estacional SMA, respectivamente.

El término $\varepsilon_{t} \sim$ Ruido Blanco $\left(0, \sigma^{2}\right)$ es el término de las innovaciones, $d$ es el orden de integración del proceso no estacional (número de raíces unitarias no estacionales) y D es el orden de integración del proceso estacional (número de raíces unitarias estacionales) (Greene, 2008).

Para su aplicación se utiliza por lo general la metodología de Box y Jenkins (1976) referente a las etapas de identificación, estimación, contraste y predicción.

\section{RESULTADOS CUANTITATIVOS}

\subsection{Método gráfico y tasas de crecimiento trimestre a trimestre ${ }^{13}$}

Los datos presentados en la gráfico 1 no dan señales de que en el régimen contributivo haya presencia de estacionalidad, caso contrario al gráfico 2, donde se observan picos en el cuarto trimestre de los diferentes años de análisis.

13 Se toma como aproximación a la tasa de crecimiento la diferencia de logaritmos naturales $\frac{x_{t}-x_{t-1}}{x_{t-1}} \approx \ln \left(x_{t}\right)-\ln \left(x_{t-1}\right)$. 
Gráfico 1. (a) Gasto de salud en el régimen contributivo (en millones de pesos colombianos reales, $2011 \mathrm{~T} 1=100)$ y (b) su tasa de crecimiento

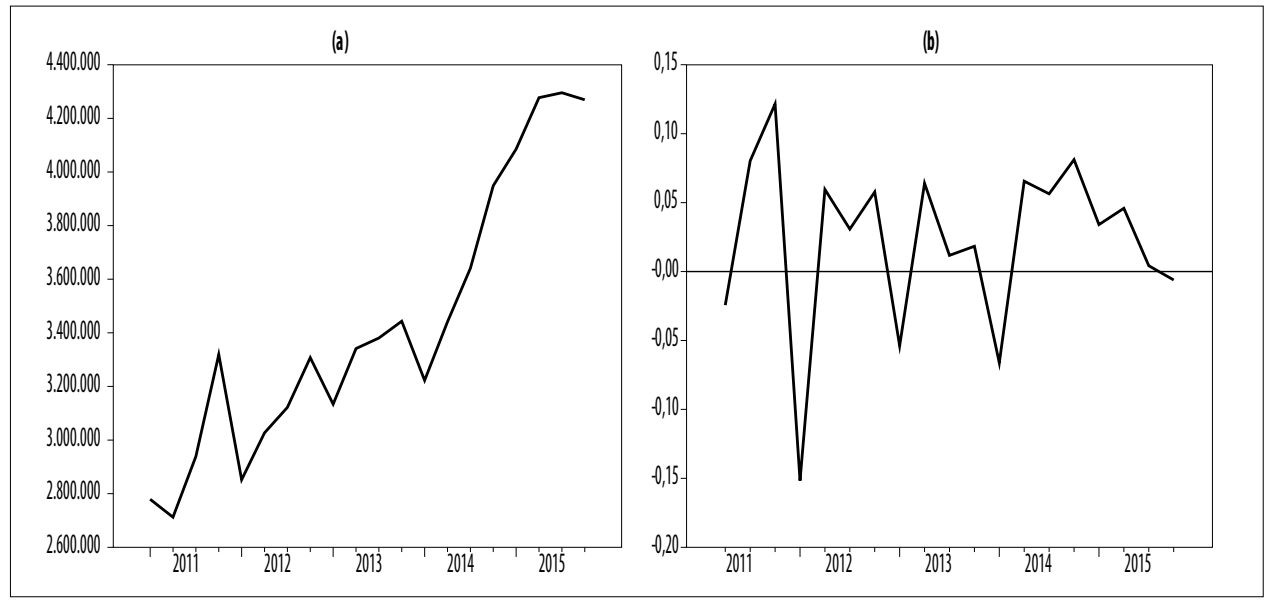

Fuente: elaboración propia

Gráfico 2. (a) Gasto de salud en el régimen subsidiado (en millones de pesos colombianos reales, $2011 \mathrm{~T} 1=100$ ) y (b) su tasa de crecimiento

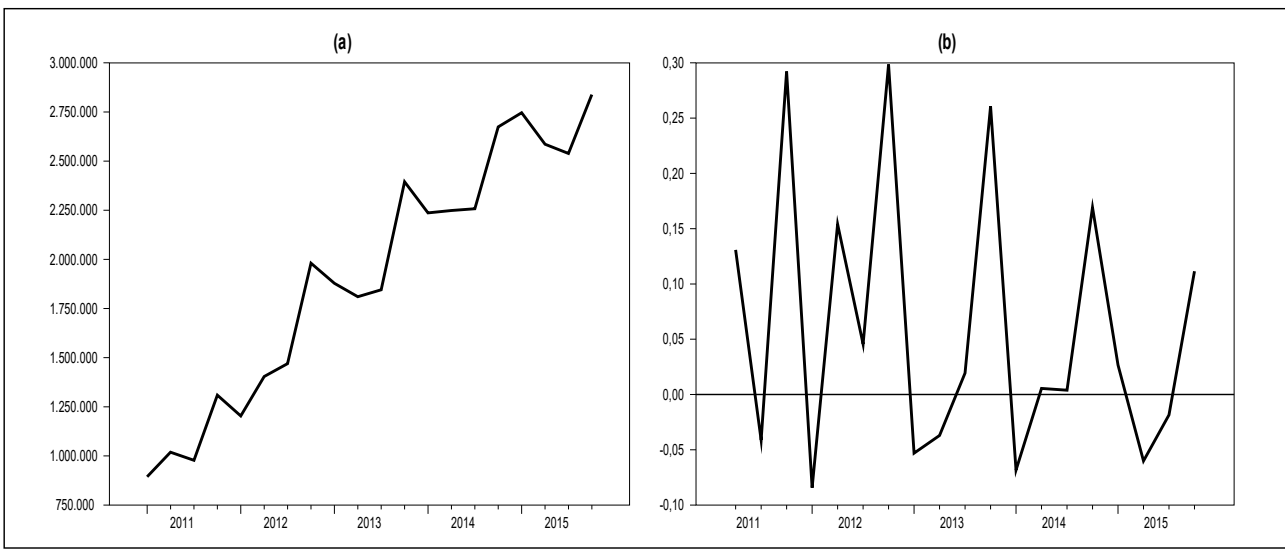

Fuente: elaboración propia

Similar comportamiento se obtiene al observar las tasas de crecimiento trimestral (tabla 1). En el régimen contributivo no hay un patrón definido, mientras que en el régimen subsidiado las tasas más altas se evidencian en el cuarto trimestre (columna T4), donde siempre se tienen crecimientos mayores al 10\%, atípicos para el resto de trimestres (T1, T2 y T3). 
Tabla 1. Tasas de crecimiento trimestral del gasto de salud (porcentajes).

\begin{tabular}{|c|c|c|c|c|c|c|c|c|c|}
\hline \multicolumn{5}{|c|}{ Régimen contributivo } & \multicolumn{5}{c|}{ Régimen subsidiado } \\
\hline & T1 & T2 & T3 & T4 & & T1 & T2 & T3 & T4 \\
\hline 2011 & & $-2,41 \%$ & $8,36 \%$ & $12,92 \%$ & 2011 & & $13,98 \%$ & $-4,04 \%$ & $33,93 \%$ \\
\hline 2012 & $-14,07 \%$ & $6,13 \%$ & $3,13 \%$ & $5,95 \%$ & 2012 & $-8,08 \%$ & $16,66 \%$ & $4,66 \%$ & $34,78 \%$ \\
\hline 2013 & $-5,24 \%$ & $6,61 \%$ & $1,18 \%$ & $1,85 \%$ & 2013 & $-5,17 \%$ & $-3,64 \%$ & $1,96 \%$ & $29,77 \%$ \\
\hline 2014 & $-6,40 \%$ & $6,78 \%$ & $5,80 \%$ & $8,46 \%$ & 2014 & $-6,61 \%$ & $0,55 \%$ & $0,39 \%$ & $18,44 \%$ \\
\hline 2015 & $3,47 \%$ & $4,69 \%$ & $0,42 \%$ & $-0,61 \%$ & 2015 & $2,70 \%$ & $-5,83 \%$ & $-1,83 \%$ & $11,80 \%$ \\
\hline
\end{tabular}

Fuente: elaboración propia

\subsection{Correlogramas simples y parciales}

La función de autocorrelación simple (FAC) y parcial (FACP) de la variable dif_ln(GSRCcont) $t_{t}$ no presenta signos de estacionalidad (gráfico 3); no obstante, la FAC de la variable dif_ $\ln (\text { GSRSubs })_{t}$ sí muestra correlaciones más altas que el promedio en los períodos 4 y 8 (gráfico 4).

Gráfico 3. (a) FAC y (b) FACP de la variable dif_In(GSRCcont)t hasta el rezago 16

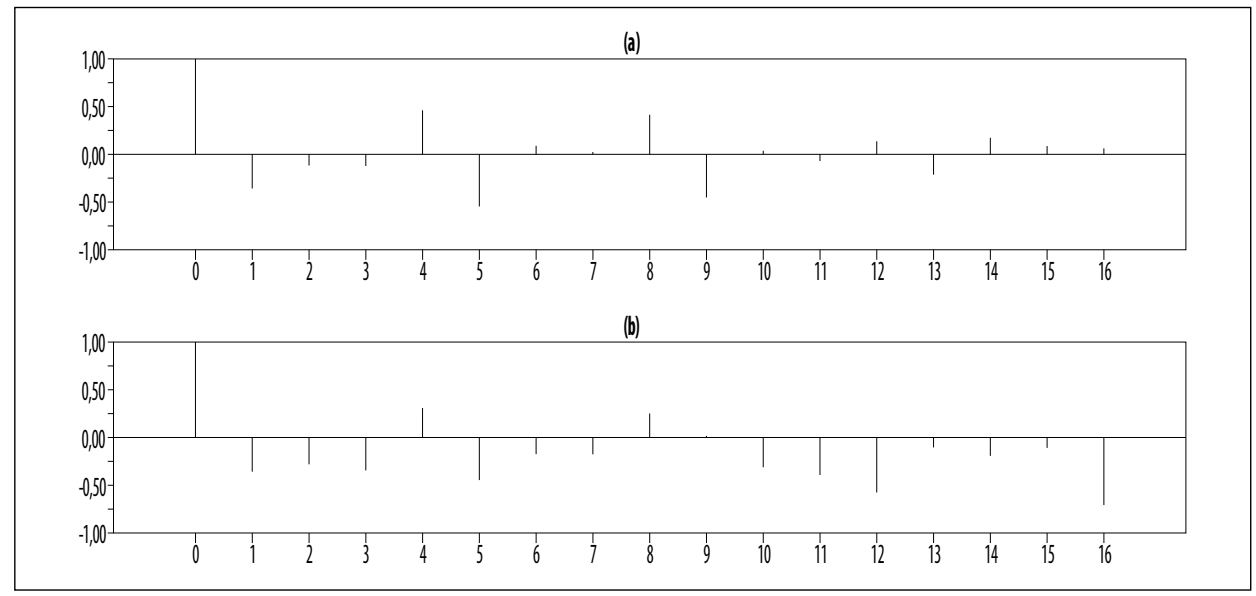

Fuente: elaboración propia 
Gráfico 4. (a) FAC y (b) FACP de la variable dif_In(GSRSubs)t hasta el rezago 16

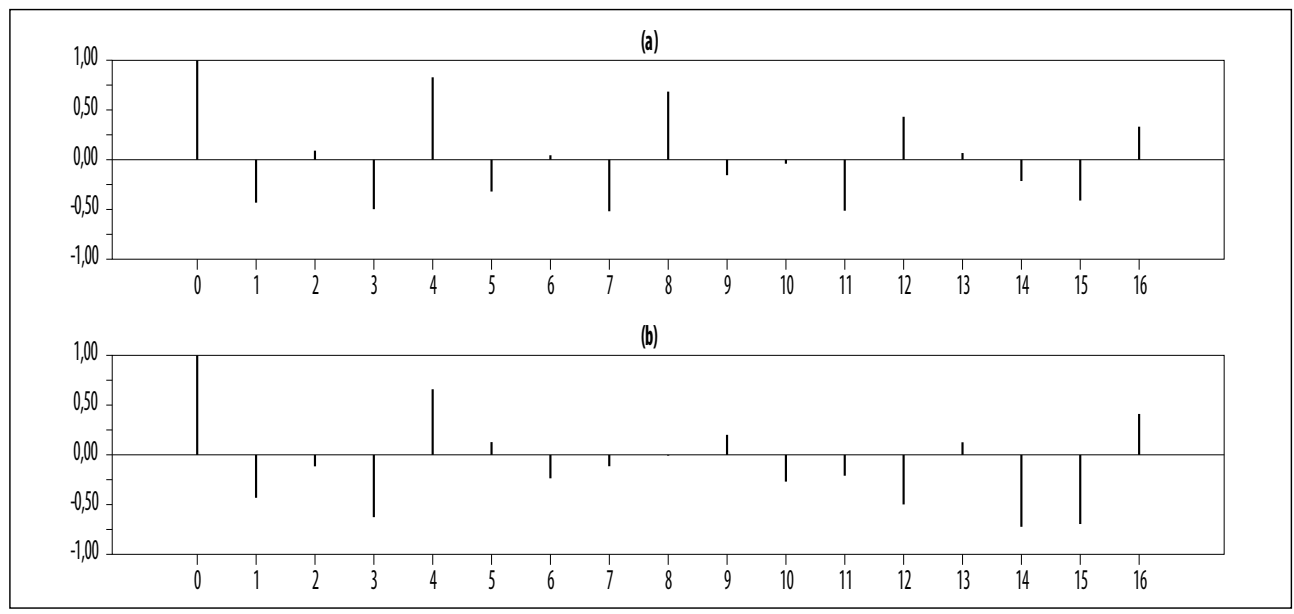

Fuente: elaboración propia

\subsection{Prueba de Kendall}

El contraste no paramétrico de Kendall arroja que para ambos casos (regímenes contributivo y subsidiado) no se rechaza la hipótesis nula de no estacionalidad, a un nivel de significancia del 5\%. Es decir que calculados los estadísticos, se establece que no hay evidencias estadísticas de componentes estacionales (tabla 2).

Tabla 2. Resultados contraste de Kendall

\begin{tabular}{|l|c|c|}
\hline & Régimen contributivo & Régimen subsidiado \\
\hline Estadístico contraste de Kendall & 3,64 & 3,08 \\
\hline Valor crítico 10\% & \multicolumn{2}{|c|}{7,81} \\
\hline
\end{tabular}

Fuente: elaboración propia

\subsection{Análisis de regresión múltiple y dummies estacionales}

Los resultados de la estimación de [8] se presentan en la tabla 3, por lo que se tiene la siguiente ecuación:

$$
\begin{aligned}
\text { GSRCont }_{t}= & 2,64 \times 10^{11}+50615,81 \text { CDSSRCont }_{t}+1,62 \times 10^{11} \mathrm{D}_{\mathrm{Q} 2} \\
& +7,93 \times 10^{10} \mathrm{D}_{\mathrm{Q} 3}+2,44 \times 10^{11} \mathrm{D}_{\mathrm{Q} 4} .
\end{aligned}
$$


Los coeficientes $\beta_{0}$ y $\beta_{1}$ son estadísticamente diferentes de cero a un alfa del $5 \%$. Aunque el coeficiente del cuarto trimestre $\left(\beta_{4}\right)$ no es significativo, es el de más alto valor.

Tabla 3. Coeficientes del modelo de regresión múltiple para el régimen contributivo ${ }^{14}$

\begin{tabular}{|c|c|c|c|c|}
\hline Variable & Coeficiente & Error estándar & Estadístico $t$ & P-valor \\
\hline$\beta_{0}$ & $2,64 \times 10^{11}$ & $5,25 \times 10^{10}$ & 5,026 & 0,000 \\
\hline$\beta_{1}$ & 50615,81 & 6406,316 & 7,901 & 0,000 \\
\hline$\beta_{2}$ & $1,62 \times 10^{11}$ & $3,51 \times 10^{11}$ & 0,461 & 0,653 \\
\hline$\beta_{3}$ & $7,93 \times 10^{10}$ & $2,94 \times 10^{11}$ & 0,270 & 0,792 \\
\hline$\beta_{4}$ & $2,44 \times 10^{11}$ & $2,87 \times 10^{11}$ & 0,850 & 0,411 \\
\hline
\end{tabular}

Fuente: elaboración propia

Los resultados de la estimación de [9] se presentan en la tabla 4. De manera que:

$$
\begin{aligned}
\text { GSRSubs }_{t}= & 2,74 \times 10^{11}+59593,40 \text { CDSSRSubs }_{t}-1,53 \times 10^{11} \mathrm{D}_{\mathrm{Q} 2} \\
& -4,60 \times 10^{11} \mathrm{D}_{\mathrm{O} 3}+9,97 \times 10^{10} \mathrm{D}_{\mathrm{O} 4} .
\end{aligned}
$$

Referente a la estimación de los parámetros de la ecuación [9] (tabla 4), se puede observar que el coeficiente $\propto_{1}$ es el único estadísticamente diferente de cero a un alfa del 5\%; no obstante, aunque el coeficiente del cuarto trimestre $\left(\propto_{4}\right)$ no es significativo, sí es cierto que es el de más alto valor, por encima de los parámetros del segundo $\left(\propto_{2}\right)$ y tercer $\left(\propto_{3}\right)$ trimestres.

Tabla 4. Coeficientes del modelo de regresión múltiple para el régimen subsidiado

\begin{tabular}{|c|c|c|c|c|}
\hline Variable & Coeficiente & Error estándar & Estadístico $t$ & P-valor \\
\hline$\propto_{0}$ & $2,74 \times 10^{11}$ & $3,13 \times 10^{11}$ & 0,876 & 0,397 \\
\hline$\propto_{1}$ & 59593,40 & 8906,228 & 6,691 & 0,000 \\
\hline$\propto_{2}$ & $-1,53 \times 10^{11}$ & $2,63 \times 10^{11}$ & $-0,582$ & 0,571 \\
\hline$\propto_{3}$ & $-4.60 \times 10^{11}$ & $2,62 \times 10^{11}$ & $-1,752$ & 0,103 \\
\hline$\propto_{4}$ & $9,97 \times 10^{10}$ & $3,59 \times 10^{11}$ & 0,278 & 0,785 \\
\hline
\end{tabular}

Fuente: elaboración propia

14 Para los análisis de regresión de los dos regímenes se tomó el período 2011T1-2015T2. 
En la tabla 5 se presentan todas las pruebas sobre los residuales de los modelos de regresión estimados, donde se observa un buen comportamiento, al no rechazar las hipótesis nulas de normalidad, no autocorrelación y homocedasticidad, en un nivel de significancia del 5\%.

Tabla 5. Validación de los residuales de los modelos de regresión múltiple

\begin{tabular}{|l|c|c|}
\hline \multirow{2}{*}{\multicolumn{1}{|c|}{ Prueba }} & $\begin{array}{c}\text { Modelo régimen } \\
\text { contributivo }\end{array}$ & \multicolumn{1}{c|}{$\begin{array}{c}\text { Modelo régimen } \\
\text { subsidiado }\end{array}$} \\
\cline { 2 - 3 } & \multicolumn{2}{|c|}{ P-valor } \\
\hline Jarque-Bera (Normalidad) & 0,457 & 0,737 \\
\hline Breusch-Godfrey LM (No Autocorrelación) & 0,270 & 0,804 \\
\hline Breusch-Pagan-Godfrey (Homocedasticidad) & 0,616 & 0,253 \\
\hline
\end{tabular}

Fuente: elaboración propia

\subsection{Procesos estocásticos SARIMA $(p, d, q)(P, D, Q)_{\mathrm{s}}$}

Antes de realizar el ajuste y la estimación de los modelos mediante máxima verosimilitud, se aplicaron las pruebas de raíces unitarias Dickey-Fuller Aumentada (ADF), Phillip-Perron (PP) y Kwiatkowski-Phillips-Schmidt-Shin (KPSS) a las variables $\ln (\text { GSRCont })_{t}$ y dif _ $\ln (\text { GSRSubs })_{t}$, para determinar si son estacionarias o no. La tabla 6 muestra que en un nivel de significancia del $5 \%$, para las pruebas ADF y PP se rechaza la hipótesis nula de raíz unitaria, y para la KPSS no se rechaza la hipótesis nula de estacionariedad.

Tabla 6. Pruebas de raíz unitaria ADF, PP y KPSS

\begin{tabular}{|c|c|c|c|c|}
\hline & \multicolumn{2}{|c|}{ Variable dif_ln(GSRCont $)_{r}$} & \multicolumn{2}{c|}{ Variable dif_ln(GSRSubs $)_{r}$} \\
\hline Test & P-valor & ¿Estacionario? & P-valor & ¿Estacionario? \\
\hline ADF & 0,044 & Sí & 0,000 & Sí \\
\hline PP & 0,000 & Sí & 0,000 & Sí \\
\hline KPSS & 0,500 & Sí & 0,290 & Sí \\
\hline
\end{tabular}

Fuente: elaboración propia

Para el caso del régimen contributivo, basándose en los criterios de información Akaike y BIC, y en el buen comportamiento de los residuales, se logró ajustar un modelo Arima $(7,1,3)$ para la serie $\ln (\text { GSRCont })_{t}$, estimando solo los coeficientes $\phi_{1}$, $\phi_{4}, \phi_{7 y} \theta_{3}$ (tabla 7). Todos los coeficientes son significativos a un alfa del $5 \%$, menos $\phi_{7}$, el cual se hace necesario incluir para tener unos residuales no correlacionados. No hubo necesidad de contar con parte estacional para la modelación. 
Tabla 7. Estimación de coeficientes del proceso ARIMA(7,1,3) para In(GSRCont)t

\begin{tabular}{|c|c|c|c|c|}
\hline Variable & Coeficiente & Error estándar & Estadístico $t$ & P-valor \\
\hline$\phi_{1}$ & 0,481 & 0,114 & 4,199 & 0,003 \\
\hline$\phi_{4}$ & 0,501 & 0,177 & 2,824 & 0,022 \\
\hline$\phi_{7}$ & 0,155 & 0,103 & 1,509 & 0,170 \\
\hline$\theta_{3}$ & $-1,014$ & 0,250 & $-4,053$ & 0,004 \\
\hline
\end{tabular}

Fuente: elaboración propia

La tabla 8 evidencia el comportamiento de ruido blanco de los residuales del modelo ARIMA $(7,1,3)$ para la serie $\ln (\mathrm{GSRC} \text { ont })_{\text {, }}$ no rechazando las hipótesis nulas de normalidad y de no autocorrelación. Al calcular la prueba no paramétrica CUSUM que tiene por hipótesis nula la buena especificación del modelo, en un nivel de confianza del 95\%, se observa que estos presentan un comportamiento adecuado (gráfico 5).

Tabla 8. Validación de los residuales del proceso ARIMA $(7,1,3)$ para In(GSRCont) ${ }_{t \prime}$

\begin{tabular}{|l|l|}
\hline \multicolumn{1}{|c|}{ Prueba } & \multicolumn{1}{c|}{ P-valor } \\
\hline Jarque-Bera (Normalidad) & 0,488 \\
\hline Ljung-Box (No Autocorrelación) & 0,010 \\
\hline
\end{tabular}

Fuente: elaboración propia

Gráfico 5. Prueba no paramétrica de especificación CUSUM sobre los residuales del proceso ARIMA $(7,1,3)$ para In $(\text { GSRCont })_{t}$

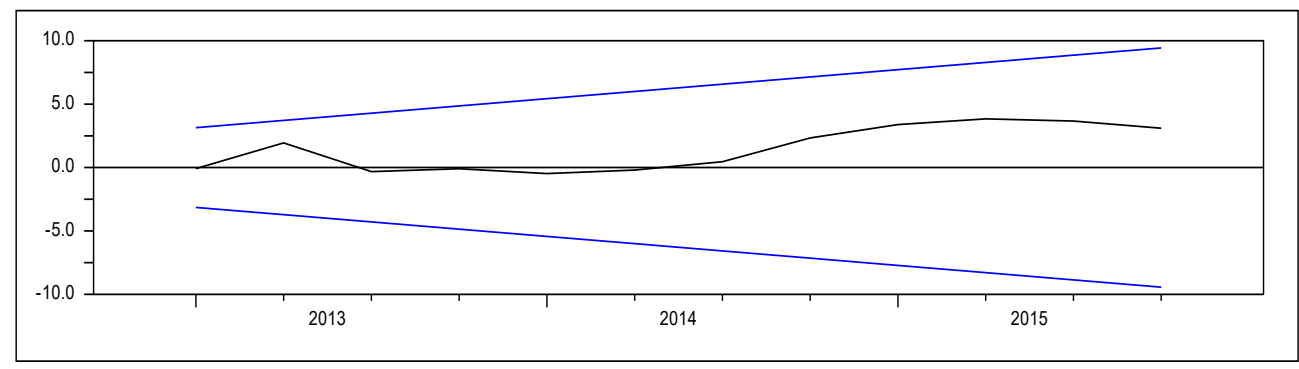

Fuente: elaboración propia

Por otra parte, para el caso del régimen subsidiado, basándose en los criterios de información Akaike y BIC, y en el buen comportamiento de los residuales, se logró ajustar un modelo SARIMA $(0,1,2)(1,0,0)_{s}$ para la serie $\ln (\mathrm{GSRSubs})_{t}$ (tabla 9). Todos los coeficientes son significativos a cualquier nivel de significancia común. 
Para estos datos, fue necesario incluir el componente estacional en el modelo de series temporales ${ }^{15}$.

Tabla 9. Estimación de coeficientes del proceso SARIMA $(0,1,2)(1,0,0)$ s para $\operatorname{In}(G S R S u b s)_{t}$

\begin{tabular}{|c|c|c|c|c|}
\hline Variable & Coeficiente & Error estándar & Estadístico $t$ & P-valor \\
\hline$\theta_{1}$ & 1,182 & 0,486 & 2,432 & 0,032 \\
\hline$\theta_{2}$ & $-1,888$ & 0,723 & $-2,612$ & 0,023 \\
\hline$\phi_{1}^{s}$ & 0,874 & 0,064 & 13,580 & 0,000 \\
\hline
\end{tabular}

Fuente: elaboración propia

De otro lado, la tabla 10 y el gráfico 6 muestran un correcto comportamiento en los residuales para la serie correspondiente al gasto del régimen subsidiado.

Tabla 10. Validación de los residuales del proceso SARIMA $(0,1,2)(1,0,0)$ s para $\operatorname{In}(G S R S u b s)_{t}$

\begin{tabular}{|l|c|}
\hline \multicolumn{1}{|c|}{ Prueba } & P-valor \\
\hline Jarque-Bera (Normalidad) & 0,111 \\
\hline Ljung-Box (No Autocorrelación) & 0,120 \\
\hline
\end{tabular}

Fuente: elaboración propia

Gráfico 6. Prueba no paramétrica de especificación CUSUM sobre residuales del proceso SARIMA $(0,1,2)(1,0,0)$ s para $\operatorname{In}(\mathrm{GSRSubs}) \mathrm{t}$

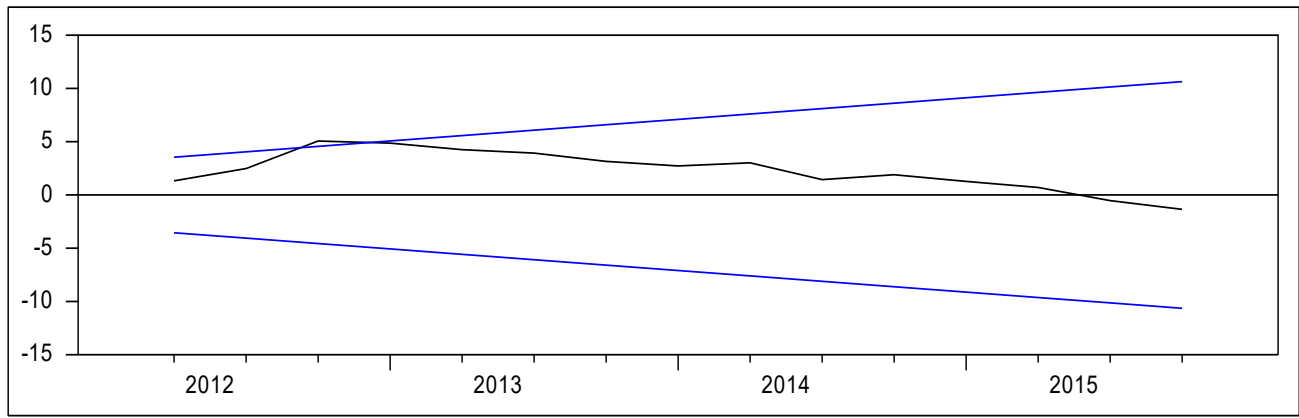

Fuente: elaboración propia

Asimismo, a través de la metodología Tramo-Seats (Gómez y Maravall, 1996) se confirma la existencia de estacionalidad en la serie de interés dado que el mejor ajuste se obtiene a partir de un $\operatorname{SARIMA}(3,1,1)$ $(0,1,1)$. No obstante, se elige el modelo estimado en la tabla 4 , dado que es más parsimonioso y sus residuales cumplen con condiciones de ruido blanco. 


\section{CONCLUSIONES}

En este estudio evalúa si el gasto en salud de los regímenes contributivo y subsidiado se comporta estacionalmente. Para ello se revisan cinco diferentes procedimientos cuantitativos. Para el régimen contributivo no se halla evidencia de estacionalidad. No obstante, se encuentra que en el régimen subsidiado sí se presenta evidencia estadística de tal componente (así lo expresan tres de los cinco métodos desarrollados), específicamente en su cuarto trimestre, período temporal en el que se observa un crecimiento mayor al de los otros trimestres (tabla 11).

Tabla 11. Resumen de evidencias sobre estacionalidad

\begin{tabular}{|l|l|l|}
\hline \multirow{2}{*}{\multicolumn{1}{c|}{ Pruebas }} & \multicolumn{2}{c|}{ ¿Hay evidencia de estacionalidad? } \\
\cline { 2 - 3 } & \multicolumn{1}{c|}{ Régimen contributivo } & \multicolumn{1}{c|}{ Régimen subsidiado } \\
\hline Gráfica y tasas de crecimiento & No & Sí \\
\hline Correlogramas simples y parciales & No & Sí \\
\hline Contraste no paramétrico de Kendall & No & No \\
\hline Regresión múltiple con dummies estacionales & No & No \\
\hline Proceso SARIMA & No & Sí \\
\hline \multicolumn{1}{|c|}{ Decisión } & $\begin{array}{c}\text { No hay evidencia } \\
\text { estadística de } \\
\text { estacionalidad }\end{array}$ & $\begin{array}{c}\text { Hay evidencia } \\
\text { estadística de } \\
\text { estacionalidad }\end{array}$ \\
\hline
\end{tabular}

Fuente: elaboración propia

Al obtener estos resultados, se procedió a indagar al detalle cuáles eran las cuentas financieras con mayor variación en su cuarto trimestre (T4), y se encontró que son las relacionadas con Contratos por evento (N. $\left.{ }^{\circ} 616540\right)$ y Contratos por eventos - Subsidiado (N. ${ }^{\circ}$ 640208), rubros que evidencian los pagos que se realizan por las actividades, procedimientos, intervenciones, insumos y medicamentos prestados o suministrados a un paciente ligado a un evento de atención en salud.

Este hecho parece tener su fundamento, entre otras razones, en el aumento de los servicios demandados en los meses finales del $a n ̃ \mathrm{O}^{16}$, dado que al aumentar el número de estas unidades de pago (constituidas por cada actividad, procedimiento, intervención, insumo o medicamento prestado o suministrado, con unas tarifas pactadas previamente), el gasto tiende a incrementar significativamente.

16 En casi todos los años de estudio el cuarto trimestre es el que presenta mayor número de servicios demandados. Ello, debido posiblemente a las enfermedades estacionales causadas por el aumento de precipitaciones en los meses de octubre y noviembre, y al efecto de las vacaciones (tiempo libre para los chequeos médicos). 
Otro aumento importante, aunque solo referente al último año de análisis (2015), es el relacionado con la cuenta Seguridad social en salud (N. ${ }^{\circ}$ 616565). Ello, debido a la implementación por parte de las EPS subsidiadas de lo dispuesto en el Decreto 2702 de 2014 del Ministerio de Salud y Protección Social, donde se establecen las directrices para la constitución de sus reservas técnicas.

De esta manera, el presente artículo de investigación muestra la importancia de entender la estacionalidad estadística a partir de los cambios regulares a través del tiempo, aportando así, conocimientos a la predicción de tendencias (patrones de conducta) respecto al flujo monetario, desde una visión macroeconómica del gasto en salud para Colombia. En unos años, con series de longitud más extensas, nuevos trabajos podrán construir modelos de pronósticos que incorporen el concepto de estacionalidad dentro de sus características intrínsecas.

Para próximas investigaciones, se deberá estudiar la dinámica del gasto en salud de las entidades promotoras de salud indígenas (que no fueron tenidas en cuenta para este documento), las cuales en la actualidad no cuentan con sistemas de información de calidad y la información que presentan en sus reportes evidencian esta debilidad. Adicionalmente, en el corto plazo será beneficioso analizar cuantitativamente el comportamiento de todos los gastos referentes al concepto de reservas, tema aún no consolidado por completo en la estructura financiera de las EPS (especialmente en las del régimen subsidiado).

\section{BIBLIOGRAFÍA}

Albert, H.; Theodoor, J.; van Bodegom, D. y Johannes, R. (2015). Seasonal variation in mortality, medical care expenditure and institutionalization in older people: Evidence from a Dutch cohort of older health insurance clients. En: PLoS One, Vol. 10, No. 11. https://doi.org/10.1371/ journal.pone.0143154.

Albouy, V.; Davezies, L. y Debrand, T. (2010). Health expenditure models: A comparison using panel data. En: Economic Modelling, Vol. 27, No. 4, p. 791-803.

Box, G. y Jenkins, G. (1976). Time series analysis: Forecasting and control. Holden Day: San Francisco, EE.UU, 575p.

Brockwell, P. y Davis, R. (2002). Introduction to time series and forecasting (2nd Ed.). Springer-Verlag: Harrisonburg, EE.UU., 437p.

Castillo-Laborde, C. y Villalobos, P. (2013). Caracterización del gasto de bolsillo en salud en Chile: Una mirada a dos sistemas de protección. En: Revista Médica de Chile, Vol. 141, No. 11, p. 1456 1463. http://dx.doi.org/10.4067/S0034-98872013001100013.

Clements, B.; Faircloth, C. y Verhoeven, M. (2007). Gasto público en América Latina: Tendencias y aspectos clave de política. En: Revista de la CEPAL, No. 93, p. 39-62. 
Ferrand, Y.; Kelton, C.; Guo, J.; Levy, M. y Yu, Y. (2011). Using time-series intervention analysis to understand U.S. Medicaid expenditures on antidepressant agents. En: Research in Social and Administrative Pharmacy, Vol. 7, No. 1, p. 64-80.

Gerdtham, U.; Søgaard, J.; Andersson, F. y Jönsson, B. (1992). An econometric analysis of health care expenditure: A cross-section study of the OECD countries. En: Journal of Health Economics, Vol. 11, No. 1, p. 63-84.

Gómez, V. y Maravall, A. (1996). Programs TRAMO and SEATS. Instructions for the User. Working Paper $N^{\circ}$ 9628, Servicio de Estudios, Banco de España, 128p.

Govindaraj, R.; Chellaraj, G. y Murray, C. (1997). Health expenditures in Latin America and the Caribbean. En: Social Science \& Medicine, Vol. 44, No. 2, p. 157-169.

Greene, W. (2008). Econometric analysis (6th Ed.). Prentice Hall: New Jersey, EE.UU, 1178p.

Hansen, P. y King, A. (1996). The determinants of health care expenditure: A cointegration approach. En: Journal of Health Economics, Vol. 15, No. 1, p. 127-137.

Hitiris, T. y Posnett, J. (1992). The determinants and effects of health expenditure in developed countries. En: Journal of Health Economics, Vol. 11, No. 2, p. 173-181.

Ke, X.; Saksena, P. y Holly, A. (2011). The determinants of health expenditure: A country-level panel data analysis. Working paper, World Health Organization, 26 p.

Kendall, M. y Ord, K. (1990). Time series (3rd Ed.). Edward Arnold: Londres, Inglaterra, 110p.

Loh, C. (2015). Trends and structural shifts in health tourism: Evidence from seasonal time-series data on health-related travel spending by Canada during 1970-2010. En: Social Science and Medicine, Vol. 132, p. 173-180. doi: 10.1016/j.socscimed.2015.03.036.

MinSalud -Ministerio de Salud y Protección Social - (2014). Cifras financieras del sector salud. Gasto en salud de Colombia: 2004-2011. En: Boletín bimestral No. 2, Dirección de Financiamiento Sectorial, 21p.

Murthy, V. y Okunade, A. (2016). Determinants of U.S. health expenditure: Evidence from autoregressive distributed lag (ARDL) approach to cointegration. En: Economic Modelling, Vol. 59, p. 67-73. https://doi.org/10.1016/j.econmod.2016.07.001.

O’Meara, G.; Ruiz, F. y Amaya, J. (2003). Impacto del aseguramiento sobre uso y gasto en salud en Colombia. Pontificia Universidad Javeriana: Bogotá, D.C., Colombia, 337p.

Pérez-Valbuena, G. y Silva-Ureña, A. (2015). Una mirada a los gastos de bolsillo en salud para Colombia. Documentos de Trabajo sobre Economía Regional, Banco de la República, $\mathrm{N}^{\circ}$ 218, 58p.

Safaeian, L.; Mahdanian, A.; Salami, S.; Pakmehr, F. y Mansourian, M. (2015). Seasonality and physician-related factors associated with antibiotic prescribing: A cross-sectional study in Isfahan, Iran. En: International Journal of Preventive Medicine, Vol. 6, No. 1. Doi: $10.4103 / 2008-7802.151 \overline{431}$.

Tang, C. (2009). An examination of the government spending and economic growth nexus for Malaysia using the leveraged bootstrap simulation approach. En: Global Economic Review, Vol. 38, No. 2, p. 215-227. 
Tsay, R. (2005). Analysis of financial time series (2nd Ed.). John Wiley E Sons, Inc.: New Jersey, EE.UU, 613p.

Yamin, A. y Parra, O. (2010). Judicial protection of the right to health in Colombia: From social demands to individual claims to public debates. En: Hastings International $\&$ Comparative Law Review, Vol. 33, No. 2, p. 431-459. 